\title{
PROMOÇÃO DA MOTRICIDADE E SAÚDE MENTAL DOS IDOSOS: UM ESTUDO DE REVISÃO
}

\section{Vera Lúcia Kerber \\ Mariana Verri Cornicelli \\ Adriana Baldo Mendes}

Mestrandos do Programa de Pós-graduação em Promoção da Saúde pelo Centro Universitário de Maringá - UNICESUMAR, Maringá (PR), Brasil.

\section{Mateus Dias Antunes}

Mestrado em andamento pelo Programa de Pós-graduação em Promoção da Saúde do Centro Universitário de Maringá - UNICESUMAR (Bolsista CAPES) e Especialização em andamento em Exercício Físico e Reabilitação do Idoso pela UNIFAMMA. Maringá (PR), Brasil.

E-mail: mateus_antunes03@hotmail.com

\section{Rute Grossi Milani}

Doutora em Ciências Medicina (Saúde Mental) pela USP, Docente do Mestrado Programa de Pós-graduação em Promoção da Saúde no Centro Universitário de Maringá - UNICESUMAR e Pesquisadora do Instituto Cesumar de Ciência, Tecnologia e Inovação - ICETI. Maringá (PR), Brasil.

\section{Sonia Maria Marques Gomes Bertolini}

Doutora em Ciências Morfofuncionais pela USP, Docente do Mestrado em Programa de Pós-graduação em Promoção da Saúde no Centro Universitário de Maringá - UNICESUMAR. Pesquisadora do Instituto Cesumar de Ciência, Tecnologia e Inovação - ICETI Maringá (PR), Brasil.
RESUMO: O objetivo deste artigo é analisar e sintetizar as informações disponibilizadas pelos estudos relevantes publicados sobre as principais práticas que estimulam a motricidade e a saúde mental dos idosos. Trata-se de uma revisão de literatura realizada com artigos indexados nas bases de dados Lilacs, Scielo, PubMed, Web of Science e Science Direct. São muitos os benefícios das atividades físicas para manutenção da mobilidade, motricidade e saúde mental dos idosos, podendo ser consideradas estratégias importantes para a promoção da saúde. Dentre as principais atividades, destacam-se na literatura o treinamento funcional, os exercícios aquáticos, a dança, as atividades nas academias da terceira idade, os exercícios físicos resistidos, a caminhada e a yoga. Nesse sentido, faz-se necessária a criação de mais espaços e maior acessibilidade aos idosos para as práticas que promovam a saúde no contexto da interdisciplinaridade, estimulando e preservando a capacidade funcional e cognitiva dessa população.

PALAVRAS-CHAVE: Atividade motora; Cognição; Envelhecimento; Promoção da saúde.

\section{PROMOTION THE MOTRICIT Y AND MENTAL HEALTH IN THE ELDERLY: A REVIEW OF THE LITERATURE}

\begin{abstract}
This study aims to analyze and summarize the information available on relevant studies published about main practices that stimulate the kinetics and mental health of elderly. This is a literature review carried out with papers indexed in the databases Lilacs, Scielo, PubMed, Web of Science and Science Direct. The studies indicated that there are many benefits of physical activities to maintain the mobility, kinetics and mental health of the elderly, and they can be considered important strategies for health promotion. The main activities include functional training, water exercises, dance, elderly gym, resistive physical exercises, walking, and yoga. Thus, it is necessary to create more spaces and accessibility for elderly for practices that promote health of this population in the context of inter-disciplinarity, stimulating and preserving the functional and cognitive capacities.
\end{abstract}

KEY WORDS: Aging, Kinetics, Cognition, Health Promotion.

\section{INTRODUÇÃO}

A motricidade é a interação de diversas funções motoras. Nesse sentido, a atividade motora tem um papel importante no desenvolvimento global do ser humano. Por meio da exploração motora, o indivíduo desenvolve a consciência de si mesmo e do mundo 
exterior; as habilidades motrizes o auxiliam na conquista de sua independência, na sua vida diária e na sua adaptação social. Os elementos básicos da motricidade humana são: motricidade fina (coordenação visuomanual quando se apanha um objeto para lançá-lo, para escrever, desenhar, pintar, entre outras) e global (movimentos dinâmicos como correr, saltar, andar, entre outros), equilíbrio, esquema corporal, organização espacial e temporal (ROSA NETO, 2009).

As modificações ocorridas com o envelhecimento não estão relacionadas apenas à questão física, mas também aos fatores psicológicos, como, por exemplo, o estresse, a depressão, problemas emocionais e a dificuldade de socialização (TAKAHASHI, 2004). A dependência física faz com que o idoso sinta-se desvalorizado, o que pode resultar no desenvolvimento de problemas psicológicos (BORGES; MOREIRA, 2009). O declínio da capacidade física, que está relacionado com o aumento da dependência do idoso, fundamenta-se na falta de mobilidade do corpo e pode conduzir o sujeito ao retraimento e à abdicação (MOREIRA, 2001).

O envelhecimento, associado à inatividade física, pode resultar em diminuição da força e da aptidão aeróbia (HICKS et al., 2012; AUYEUNG et al. 2013). A força, a capacidade cardiorrespiratória, o equilíbrio, a velocidade da marcha e os níveis de destreza manual representam uma relação positiva com o melhor desempenho das atividades diárias e maior autonomia individual (CRISTI et al., 2014).

A prática de exercícios físicos é uma das estratégias para atenuar o declínio comumente observado na aptidão funcional. Vários estudos têm demonstrado que os exercícios físicos contribuem para a melhoria da aptidão funcional (CRISTI et al., 2014; ZHUANG et al., 2014; CRUZ-FERREIRA et al., 2015).

A depressão é uma das condições mais comuns associadas ao suicídio do idoso, sendo um risco que vem crescendo, a cada dia, no mundo inteiro. Emerge como resultado de uma inibição global da pessoa, que afeta a função da mente, comprometendo o ser humano na sua totalidade, sem que seja possível fazer a separação entre o psíquico, o social e o físico, expressando sinais como o abandono da sua autoestima, o desespero em relação à vida, a tristeza profunda ou a presença constante da morte (COUTINHO et al., 2003).
Quando nos remetemos especificamente à depressão no idoso, esta é o segundo diagnóstico psiquiátrico mais frequente nas pessoas com mais idade e estima-se que $30 \%$ a $40 \%$ dos idosos apresentam algum sintoma depressivo (LEON et al., 2003). Na literatura encontram-se ações de intervenção, com base na animação sociocultural, que contribuem para a diminuição da sintomatologia depressiva, promovendo o relacionamento interpessoal e aumentando a autoestima dos idosos com depressão (COSTA, 2013).

Dessa forma, o objetivo deste estudo é analisar e sintetizar as informações disponibilizadas pelos estudos relevantes publicados sobre as principais práticas que estimulam a motricidade e a saúde mental dos idosos.

\section{METODOLOGIA}

Trata-se de uma revisão de literatura realizada por meio de uma busca de artigos publicados nacional e internacionalmente e indexados nas seguintes bases de dados: Lilacs, Scielo, PubMed, Web of Science e ScienceDirect. Foram utilizados os seguintes Descritores em Ciências da Saúde (DeCS): atividade motora, idoso, envelhecimento, destreza motora, flexibilidade, força muscular, aptidão física, exercício, humor, ansiedade, depressão e promoção da saúde.

Após a busca dos artigos com os respectivos descritores, foram selecionados de acordo com critérios de inclusão: presença de descritores escolhidos no título do trabalho ou inseridos no resumo; artigos na íntegra, disponíveis na internet, idiomas português, inglês e espanhol, e publicação de 2000 a 2017. Como critérios de exclusão, foram considerados o não preenchimento das informações no título ou resumo, os artigos estarem em mais de uma base de dados e os artigos terem sido publicados em período diferente daquele da pesquisa. A busca de dados foi realizada no período de maio a junho de 2017

\section{PROMOÇÃO DA MOTRICIDADE EM IDOSOS}

Foi possível verificar diversas possibilidades de modalidades de atividades físicas que promovem 
a melhoria dos elementos básicos da motricidade dos idosos.

Em um estudo realizado por Ferreira e Gobbi (2003) visando verificar a influência do treinamento com atividades físicas generalizadas e supervisionadas, na agilidade geral e na agilidade de membros superiores de 60 idosas, elas foram divididas em 2 grupos: as que participavam de um grupo supervisionado de atividades físicas generalizadas e aquelas que faziam parte do grupo controle, constituído por idosas que não realizavam atividades físicas. Após a avaliação dos testes de agilidade, foi possível encontrar diferenças estatisticamente significativas entre os grupos para o teste de agilidade geral. No entanto, não foram encontradas diferenças na agilidade de membros superiores.

A agilidade é a capacidade que o indivíduo tem de realizar movimentos rápidos com mudança de sentido e direção e seus principais fatores que influenciam são: força, velocidade, flexibilidade e coordenação motora (SANTOS et al., 2015). Esta capacidade é exigida na maioria das atividades do dia-a-dia do idoso, como andar desviando de outros obstáculos e pessoas, locomover-se carregando abjetos e andar rapidamente para atravessar a rua, para atender um telefone ou campainha (SILVA et al., 2002). Isto posto, manter bons níveis de agilidade contribui para a prevenção de quedas, fato muito comum entre os idosos e que representa uma das principais causas de morbidade e mortalidade por causas externas nesta população, tornando-se um importante problema de saúde pública devido aos altos custos financeiros e sociais (GUERRA et al., 2017).

No período de 24 semanas, com 3 visitas por semana, em dias não consecutivos, Silva et al. (2008) avaliaram o equilíbrio, a coordenação e a agilidade de 61 idosos submetidos a treinamento de força com carga progressiva e, após a intervenção, pode-se verificar que o programa de treinamento de força mostrou-se favorável na melhoria do desempenho funcional e motor de idosos ao comparar com um grupo controle que não realizou treinamento de força.

Os exercícios realizados no ambiente aquático, como a natação e a hidroginástica, vêm destacando-se pela conquista cada vez maior de adeptos (GONÇALVES et al., 2014) e apresentando evidências científicas dos seus benefícios terapêuticos para idosos (LIMA et al., 2017). A propriedade de sustentação da água (empuxo) alivia o estresse sobre as articulações que sustentam o peso corporal, auxiliando no equilíbrio estático e dinâmico (FUJISHIMA; SHIMIZU, 2003). Desta maneira, proporciona maior facilidade na realização de movimentos que seriam difíceis ou impossíveis de serem realizados no solo sem auxilio (FUJISHIMA; SHIMIZU, 2003). Além de recomendadas pelos médicos, estudos evidenciam que a prática regular de atividades aquáticas é adequada à terceira idade, pois proporciona segurança ao idoso, auxilia na postura e na mobilidade, melhora a aptidão física e as atividades diárias, bem como facilita a socialização dos idosos (OLIVEIRA et al., 2017).

Em relação à natação, encontrou-se na literatura um estudo de Rabelo et al. (2008) visando verificar o seu efeito sobre a realização das atividades de vida diária de 44 idosas, divididas em dois grupos, sendo o controle $(n=20)$ composto por mulheres que não realizaram nenhuma atividade física e o teste $(\mathrm{n}=24)$ composto por mulheres que foram as realizaram por um período de 12 semanas, três vezes por semana, cada sessão de natação com duração de 50 minutos. Após a intervenção foi verificada melhora significativa nas variáveis da capacidade funcional. Já o grupo teste não apresentou nenhuma melhora significativa. Deste modo, considerouse a natação como uma estratégia favorável de prática de atividade física para melhora das capacidades de realização das atividades de vida diária.

Alves et al. (2014) realizaram um estudo com intuito de verificar o efeito da prática de hidroginástica sobre a aptidão física de 74 idosas, separadas em grupo controle $(n=37)$ e grupo intervenção, que realizou duas aulas semanais de hidroginástica durante três meses. Constatou-se que, após a intervenção, o grupo intervenção apresentou melhor desempenho nos componente da aptidão física quando comparado com os resultados do grupo controle. Outros estudos também evidenciam a hidroginástica como uma atividade física efetiva para melhora do condicionamento cardiorrespiratório (KANITZ et al., 2015; SCHOENELL; BGEGINSKI; KRUEL, 2017) e qualidade de vida (RICA et al., 2013). Assim, a prática regular de hidroginástica estabelece uma relação com uma via saudável, resultando 
em melhorias fisiológicas, psicológicas e sociais (PINTO; DIAS; MOREIRA, 2014).

As academias da terceira idade também são consideradas espaços e práticas que estimulam a saúde e a inserção social dos idosos e, consequentemente, promovem melhoria significativa na saúde física e mental (COSTA; FREITAS; SILVA, 2016). Após sua implantação em 2006 no município de Maringá - PR (PALÁCIOS; NARDI, 2007), estudos vêm sendo realizados para verificar o impacto nas capacidades funcionais dos idosos (ESTEVES et al., 2011; MALTA et al., 2014). Gozzi, Bertolini e Lucena (2016) realizaram um estudo com 600 idosos, sendo 300 praticantes de exercícios físicos nas academias da terceira idade, e 300 idosos não praticantes, o qual constatou que os idosos praticantes de exercícios físicos nas academias da terceira idade têm maior agilidade e capacidade cognitiva e melhor equilíbrio, quando comparados aos não praticantes.

Dentre outras oportunidades de promoção de práticas corporais e atividades físicas, há a dança sênior, uma atividade lúdica e intrinsecamente motivante que previne a inatividade e retarda os processos da senilidade, melhora a qualidade de vida e otimiza a socialização (SANTOS et al., 2016). Gouvêa et al. (2017) verificaram os efeitos da dança sênior sobre os parâmetros motores, cognitivos e na qualidade de vida de idosos após um programa de atividade física de dança sênior pelo um período de três meses, no qual verificou-se que a prática foi efetiva na saúde física e mental e na qualidade de vida dos longevos. Nota-se na literatura variados estudos que utilizam a dança sênior como uma intervenção para melhoria da postura e equilíbrio (KATTENSTROTH et al., 2010), mobilidade funcional (HACKNEY; KANTOROVICH; EARHART, 2007), redução das quedas (HEIBERGER et al., 2011), qualidade de vida (GOUVÊA et al., 2017) e estado de saúde (HUI; CHI; WOO, 2009).

Ao longo dos anos, vem destacando-se outra modalidade de atividades física, o treinamento funcional. $\mathrm{O}$ mesmo visa melhorar a aptidão de seus praticantes para realização das funções que são necessárias em seu cotidiano (LUSTOSA et al., 2010). No estudo de Miranda et al. (2016) foi avaliado o efeito de 9 semanas de treino funcional sobre os índices de aptidão neuromuscular de idosas por meio de um programa que foi aplicado três vezes por semana durante o período supracitado. As sessões tiveram duração de 45 minutos e logo após a intervenção foi possível verificar melhora da força e resistências das idosas. Emídio et al. (2017) analisaram os efeitos positivos do treinamento funcional na melhora das capacidades neuromotoras dos idosos o que, por consequente, melhorou a funcionalidade nas atividades de vida diária e qualidade de vida dos idosos.

Não obstante, Pereira et al. (2017) verificaram a influência da aplicação de um protocolo de 24 sessões, com frequência de 3 vezes, na semana de treinamento funcional sobre a funcionalidade e equilíbrio de idosos não institucionalizados. O protocolo foi dividido em quatro fases: aquecimento, exercícios de equilíbrio, fortalecimento muscular e alongamentos. Ao final da intervenção verificou-se melhora no equilíbrio estático e dinâmico dos idosos o que, consequentemente, favoreceu o declínio do risco de quedas. Assim, o treinamento funcional está baseado na melhoria dos aspectos neurológicos que afetam a capacidade funcional do corpo humano por meio de exercícios que desafiam os componentes do sistema nervoso e (RESENDE NETO et al., 2016) e, por isso, estimulam sua adaptação (ALMEIDA; SILVA, 2016).

\section{PROMOÇÃO DA SAÚDE MENTAL EM IDOSOS}

O envelhecimento não pode ser considerado uma enfermidade incapacitante, é preciso adotar atitudes positivas. É importante que o idoso mantenha-se em uma ocupação do seu interesse, que exercite a mente e, se possível, que tenha remuneração adequada para que se sinta valorizado. No que diz respeito à afetividade, fazse necessário mantê-lo sempre próximo e incentivá-lo a demonstrar seus sentimentos (PAPALÉONETTO, 2002).

Portanto, o envelhecimento é um processo que deve ser vivenciado com autonomia, reconhecimento de direitos, segurança, dignidade, bem-estar e saúde. Ações de promoção da saúde mental em idosos são primordiais na redução do surgimento de transtornos como depressão, ansiedade e suicídio, pois são úteis no desenvolvimento de habilidades como empoderamento, autonomia e autoeficácia (GIARDINI, 2014). 
Dessa forma, a Organização Mundial da Saúde (OMS) relata a saúde mental como um estado de bem-estar no qual as pessoas acreditam em suas potencialidades e capacidades de lidar com as dificuldades do dia-a-dia, trabalhando de forma positiva e contribuindo para o crescimento da sua comunidade. Assim, a inserção de intervenções promotoras de saúde é fundamental para a saúde mental da população mundial (BRASIL, 2006).

No que se refere ao foco de intervenções de promoção à saúde mental de idosos, ressalta-se a importância das intervenções para o empoderamento dessas pessoas a partir de encontros em grupos que têm como intuito promover a valorização do envelhecer e a discussão de questões referentes à longevidade. Existem evidências de que uma abordagem de empoderamento é uma perspectiva de autoeficácia e que esta pode impulsionar a participação do idoso nas decisões de saúde e promover resultados positivos para a sua vida, tendo como resultado a redução da depressão e ansiedade e o fortalecimento da autoeficácia dos participantes (SHEARER et al., 2012).

No mesmo sentido, Teixeira et al. (2016) mostram resultados eficientes quanto à promoção da saúde em idosos praticantes de atividade física. $\mathrm{O}$ estudo evidencia que a prática de atividades físicas aumenta os níveis de autoestima e reduz os níveis de depressão. Constatouse que a autoestima diminui com a idade, enquanto os sintomas depressivos aumentam.

As práticas corporais mais relatadas nos documentos publicados pelo Ministério da Saúde que podem ser realizadas em grupos de idosos da atenção primária à saúde consistem em caminhada, danças, e yoga. Esta última é definida como prática corporal oriunda da medicina tradicional chinesa que visa trazer equilíbrio entre corpo e mente, diminuindo a ansiedade e o estresse causado pelo cotidiano (BRASIL, 2006; HOWE et al., 2011).

Desse modo, as práticas relatadas atuam na manutenção da capacidade funcional do idoso. Os benefícios dessas práticas favorecem os aspectos biopsicossociais. Dentre eles, destacam-se a preservação da independência, a redução da ansiedade e do estresse e a melhora do estado de humor. As atividades devem ser prazerosas, de intensidade moderada, baixo impacto, curto tempo ( $30 \mathrm{a} 45 \mathrm{~min}$ ) e de três a quatro vezes por semana (BRASIL, 2006).

No caso dos idosos que têm dificuldade de contato corporal com outros participantes, pode-se pensar na realização de caminhadas associadas a práticas corporais oriundas da medicina tradicional chinesa que contribuam para o equilíbrio entre corpo e mente e sejam capaz de reduzir a ansiedade, e melhorar o estado de humor dos idosos praticantes. O incentivo dessas práticas nas unidades de saúde, além de trazer os benefícios citados de prevenção e promoção da saúde aos usuários, favorece a interdisciplinaridade e a troca de saberes especialmente entre os profissionais, oferecendo melhor qualidade de vida a população idosa (BRASIL, 2006 ; HOWE et al., 2011).

$\mathrm{Na}$ intenção de relacionar o nível de atividade física com saúde mental, um estudo de base populacional com amostragem de 875 idosos da cidade de Florianópolis, mostrou que o nível de atividade física baixo relaciona-se com uma pior saúde mental em idosos (BENEDETTI et al., 2008). Outro estudo comparativo dos níveis de ansiedade e depressão entre os idosos sedentários e ativos revelou maiores índices de ansiedade e depressão em idosos sedentários, ou seja, verificou-se que o exercício físico promove uma redução nos sintomas de ansiedade e depressão (MINGHELLI, 2013).

Assim, diante da importância da atividade física na promoção da saúde, do ponto de vista biológico a atividade física tem sido associada a vários fatores favoráveis a uma melhor qualidade de vida no idoso, principalmente no que tange à condição mental. A atividade física eleva a autoestima do idoso, contribui para as relações psicossociais e estimula as funções cognitivas no que diz respeito à capacidade de concentração, memória e melhora do quadro de depressão (STELLA et al., 2002).

\section{CONCLUSÃo}

Conclui-se que são muitos os benefícios das atividades físicas para manutenção da mobilidade, motricidade e saúde mental dos idosos, podendo, assim, serem consideradas importantes estratégias para 
promoção da saúde. Dentre as principais atividades, destacam-se na literatura o treinamento funcional, os exercícios aquáticos, a dança, as atividades nas academias da terceira idade, os exercícios físicos resistidos, a caminhada, a yoga. Nesse sentido, faz-se necessária a criação de mais espaços e maior acessibilidade aos idosos para as práticas que promovam a saúde no contexto da interdisciplinaridade, estimulando e preservando a capacidade funcional e cognitiva dessa população.

\section{AGRADECIMENTOS}

Ao Instituto Cesumar de Ciência, Tecnologia e Inovação - ICETI, ao Centro Universitário de Maringá UNICESUMAR e à Coordenação de Aperfeiçoamento de Pessoal de Nível Superior - CAPES pela concessão de bolsas de auxílio à pesquisa.

\section{REFERÊNCIAS}

ALMEIDA, D.K.S.; SILVA, F.O.C. A função muscular e a composição corporal na qualidade de vida do idoso: efeitos de um programa de 8 semanas de treinamento combinado. Revista Brasileira de Prescrição e Fisiologia do Exercício, v. 10, n. 60, p. 504-510, 2016.

ALVES, R.V. et al. Physical fitness andelderlyhealtheffectsofhydrogymnastics. Revista Brasileira de Medicina do Esporte, v. 10, n. 1, p. 31-37, 2014.

AUYEUNG T.W. et al. Age-associated decline ofmusclemass, gripstrengthandgaitspeed: a 4-year longitudinal studyof 3018 community-dwellingolderChinese. Geriatrics\&Gerontolog yInternational, v. 14, n. 11, p. 76-84, 2014.

BENEDETTI, T. R. B. et al. Atividade física e estado de saúde mental de idosos. Revista de Saúde Pública, v. 42, n. 2, p.302-307, 2008.

BORGES, M.R.D.; MOREIRA, A.K. Influências da prática de atividades físicas na terceira idade: estudo comparativo dos níveis de autonomia para o desempenho nas AVDs e AIVDs entre idosos ativos fisicamente e idosos sedentários.
Revista Motriz, v. 15, n. 1, p. 562-573, 2009.

BRASIL. Ministério da Saúde. Cadernos de Atenção Básica: Envelhecimento e saúde da pessoa idosa (Caderno de Atenção Básica n. 19) [recurso eletrônico]. Ministério da Saúde, Secretaria de Atenção à Saúde, Departamento de Atenção Básica. Brasília: Ministério da Saúde, 2006. Disponívelem: http://bvsms.saude.gov.br/ bvs/publicacoes/abcad19.pdf . Acesso em 06 de junho de 2017.

BRASIL. Ministério da Saúde. Política Nacional de Práticas Integrativas e Complementares no SUS. Atitude e ampliação do acesso. Ministério da Saúde, Secretaria de Atenção à Saúde, Departamento de Atenção Básica. Brasília: Ministério da Saúde, 2006. Disponível em: http:// bvsms.saude.gov.br/bvs/publicacoes/pnpic.pdf . Acesso em 06 de junho de 2017.

COSTA, B.; FREITAS, C.; SILVA, K. Atividade física e uso de equipamentos entre usuários de duas Academias ao Ar Livre. Revista Brasileira de Atividade Física \& Saúde, v. 21, n. 1, p. 29-38, 2016.

COSTA, P.I.F. Promoção da Saúde Mental em Idosos: Projeto de Intervenção na Depressão com base na Animação Sociocultural. Coimbra: Escola Superior de Tecnologia da Saúsde de Coimbra, 2013.93f. Dissertação (Mestrado em Educação para a Saúde). Programa de Pósgraduação em Educação para Saúde, Coimbra, 2013.

COUTINHO, M. et al. Depressão, um sofrimento sem fronteira: representações sociais entre crianças e idosos. Revista Psico-USF, v. 8, n, 2, p. 183-182, 2003.

CRISTI, C. et al. Whole-bodyvibration training increasesphysical fitness measureswithoutalterationofinflammatorymarkers in olderadults. EuropeanJournalof Sport Science, v. 14, n. 6, p. 611-619, 2014.

CRUZ-FERREIRAA. et al. Creative Dance Improves Physical Fitness and Life Satisfaction in OlderWomen. Researchon Aging, v. 37, n. 8, p. 837-855, 2015.

EMÍDIO, R.G. et al. Efeito do treinamento funcional na melhora das capacidades neuromotoras de idosos. Brazilian Journal of Surgery and Clinical Research, v. 
18, n. 1, p. 6-9, 2017.

ESTEVES, J.V.D.C. et al. O uso de academias da terceira idade por idosos modifica parâmetros morfofuncionais? Acta Scientiarum. Health Sciences, v. 34, n. 1, p. 31-38, 2011.

FERREIRA, L.; GOBBI, S. Agilidade geral e agilidade de membros superiores em mulheres de terceira idade treinadas e não treinadas. Revista Brasileira de Cineantropometria e Desempenho Humano, v. 5, n. 1, p. 46-53, 2003.

FUJISHIMA, K.; SHIMIZU, T. Bodytemperature, oxygenuptakeandheart rate duringwalking in waterandonlandatanexerciseintensitybasedon RPE in elderlymen. Journal of Physiological Anthropology and Applied Human Science, v. 22, n. 2, p. 83-88, 2003.

GIARDINI, S. Prevenção e promoção da saúde mental no envelhecimento: conceitos e intervenções. Psicologia: Ciência e profissão, v. 34, n. 2, p. 318-329, 2014.

GONÇALVES, K.C. et al. Comparação entre a percepção da qualidade de vida e o nível de aptidão física de idosos praticantes de atividades aquáticas. Revista de Atenção à Saúde, v. 12, n. 39, p. 35-40, 2014.

GOUVÊA, J.A.G. et al. ImpactofSenior Dance onemotionaland motor parametersandqualityoflifeoftheelderly. Northeast Network NursingJournal, v. 18, n. 1, p. 51-58, 2017.

GOZZI, S.D.; BERTOLINI, S.M.M.G.; LUCENA, T.F.R. Impacto das academias da terceira idade: comparação da capacidade motora e cognitiva entre praticantes e não praticantes. ConScientiae Saúde, v. 15, n. 1, p. 15-23, 2016.

GUERRA, H.S. et al. Prevalência de quedas em idosos na comunidade. Saúde e Pesquisa, v. 9, n. 3, p. 547-555, 2017.

HACKNEY, M.E.; KANTOROVICH, S.; EARHART, G.M. A studyontheeffectsof Argentine tango as a formofpartnered dance for thosewith Parkinson diseaseandthehealthyelderly. American Journalof Dance Therapy, v. 29, n. 2, p. 109-127, 2007.

HEIBERGER, L. et al. Impactof a weekly dance classonthe- functionalmobilityandonthequalityoflifeofindividualswithParkinson'sdisease. Frontiers in AgingNeuroscience, v. 3, p. 14, p. 1-15, 2011.

HICKS, G.E. et al. Absolutestrengthandlossofstrength as predictorsofmobility decline in olderadults: theInCHIANTIstudy. The JournalsofGerontology. Series A, BiologicalSciencesand Medical Sciences, v. 67, n. 1, p. 6673, 2012.

HOWE, T. E. et al. Exercise for improving balance in olderpeople. The Cochrane Library, v. 9, n. 11, p. 1-12, 2007.

HUI, E.; CHUI, B.T.; WOO, J. Effectsof dance onphysicalandpsychologicalwell-being in olderpersons. Archives of Gerontology and Geriatrics, v. 49, n. 1, p. 45-50, 2009.

KANITZ, A.C. et al. Effectsoftwodeepwater training programsoncardiorespiratoryand muscular strength responses in olderadults. Experimental Gerontology, v. 64, p. 55-61, 2015.

KATTENSTROTH, J.C. et al. Superior sensory, motor, andcognitive performance in elderlyindividualswithmultiyear dancing activities. Frontiers in Aging Neuroscience, v. 2, p. 31, p. 1-9, 2010.

LEON, F.G. et al. Depressionandcomorbid medical illness: Therapeuticanddiagnosticchallenges. The Journal of Family Practice, v. 19, n. 1, p. 33-43, 2003.

LIMA, V.P. et al. Variações Hemodinâmicas Em Idosas Pré E Pós-Exercícios Em Hidroginástica. Journal of Physical Education, v. 86, n. 1, p. 18-24, 2017.

LUSTOSA, L.P. et al. Efeito de um programa de treinamento funcional no equilíbrio postural de idosas da comunidade. Fisioterapia e Pesquisa, v. 17, n. 2, p. 153-156, 2010.

MALTA, D. et al. Política Nacional de Promoção da Saúde, descrição da implementação do eixo atividade física e práticas corporais, 2006 a 2014. Revista Brasileira de Atividade Física \& Saúde, v. 19, n. 3, p. 286, 2014.

MINGHELLI, B. et al. Comparação dos níveis de ansiedade e depressão entre idosos ativos e sedentários. Revista 
de Psiquiatria Clínica, v. 40, n. 2, p. 71-76, 2013.

MIRANDA, L.V. et al. Efeitos de 9 semanas de treinamento funcional sobre índices de aptidão muscular de idosas. Revista Brasileira de Prescrição e Fisiologia do Exercício, v. 10, n. 59, p. 386-394, 2016.

MOREIRA, C.A. Atividade física na maturidade: avaliação e prescrição de exercícios. Rio de Janeiro: Shape, 2001.

OLIVEIRA, D.V. et al. Functionalcapacityandqualityoflife in olderwomenpracticingandnotpracticinghydrogymnastics. Northeast Network NursingJournal, v. 18, n. 2, p. 156-163, 2017.

PALÁCIOS, A.R.O.P.; NARDI, A.C.F. Academia da Terceira Idade: promoção da saúde e atividade física em Maringá. Divulgação, Saúde e Debate, n. 40, v. 1, p. 71-76, 2007.

PAPALÉO Netto, Matheus. Gerontologia. São Paulo: Editora Atheneu, 2002.

PEREIRA, L.M. et al. Impactos do treinamento funcional no equilíbrio e funcionalidade de idosos não institucionalizados. Revista Brasileira de Ciência e Movimento, v. 25, n. 1, p. 1-10, 2017.

PINTO, L.L.T.; DIAS, I.M.; MOREIRA, R.M. Análise Reflexiva Sobre os Benefícios da Hidroginástica para a Saúde de Pessoas Idosas. Saúde e Pesquisa, v. 7, n. 2, p. 353-359, 2014.

RABELO, R.J. et al. Efeitos da natação na capacidade funcional de mulheres idosas. Revista Brasileira de Ciência e Movimento, v. 12, n. 3, p. 57-60, 2008.

RESENDE NETO, A.G. et al. Treinamento funcional para idosos: uma breve revisão. Revista Brasileira de Ciência e Movimento, v. 24, n. 3, p. 58-71, 2016.

RICA, R.L. et al. Effectsofwater-basedexercise in obeseolderwomen: Impactof short-term follow-up studyonanthropometric, functional fitness andqualityoflifeparameters. Geriatrics \& Gerontology International, v. 13, n. 1, p. 209-214, 2013.

ROSA NETO et al. Manual de avaliação motora para a terceira idade. Porto Alegre: Artmed, 2009.
SANTOS, L.A.D. Efeito da dança sênior no equilíbrio e no risco de quedas em hemiparéticospós acidente vascular encefálico. Fisioterapia Brasil, v. 14, n. 1, v. 56-60, 2016.

SANTOS, R.K.M. et al. Prevalência e fatores associados ao risco de quedas em idosos adscritos a uma Unidade Básica de Saúde do município de Natal, RN, Brasil. Revista Ciência \& Saúde Coletiva, v. 20, n. 12, p. 3753-3762, 2015.

SCHOENELL, M.; BGEGINSKI, R.; KRUEL, L. Efeitos do treinamento em meio aquático no consumo de oxigênio máximo de idosos: revisão sistemática com metanálise de ensaios clínicos randomizados. Revista Brasileira de Atividade Física \& Saúde, v. 21, n. 6, p. 525-533, 2017.

SHEARER, N. et al. Empowermentinterventions for olderadults. Western Journal of Nursing Research, v. 34, n. 1, p. 24-51, 2012.

SILVA, A. et al. Equilíbrio, coordenação e agilidade de idosos submetidos à prática de exercícios físicos resistidos. Revista Brasileira de Medicina do Esporte, v. 14, n. 2, p. 88-93, 2008.

SILVA, V.M. et al. Nível de agilidade em indivíduos entre 42 e 73 anos: efeitos de um programa de atividades físicas generalizadas de intensidade moderada. Revista Brasileira de Ciências do Esporte, v. 23, n. 3, p. 65-79, 2002.

STELLA, F. et al. Depressão no idoso: diagnóstico, tratamento e benefícios da atividade física. Motriz, v. 8, n. 3, p. 91-98, 2002.

TAKAHASHI, S.R.S. Benefícios da atividade física na melhor idade. Revista Digital, v. 10, n. 74, p. 1-10, 2004.

TEIXEIRA, C. M. et al. Physicalactivity, self-esteemanddepression in olderadults. Cuadernos de Psicologíadel deporte, v. 16, n. 3, p. 55-67, 2016.

ZHUANG, J. et a. The effectivenessof a combinedexerciseinterventiononphysical fitness factorsrelatedtofalls in community-dwellingolderadults. Clinical Interventions in Aging, v. 9, n. 1, p. 131-140, 2014.

Recebido em: 01 abril de 2017 Aceito em: 30 de agosto de 2017 